\title{
Two-stage acceleration of interstellar ions driven by high-energy lepton plasma flows
}

\author{
CUI YunQian ${ }^{1}$, SHENG ZhengMing ${ }^{2,3,4},{ }^{*}$ LU QuanMing ${ }^{5}$, LI YuTong ${ }^{1,4}$, and ZHANG Jie ${ }^{3,4}$ \\ 1 Beijing National Laboratory of Condensed Matter Physics, \\ Institute of Physics, CAS, Beijing 100190, China; \\ 2 SUPA, Department of Physics, University of Strathclyde, Glasgow G4 0NG, United Kingdom; \\ ${ }^{3}$ Key Laboratory for Laser Plasmas (MoE) and Department of Physics and Astronomy, \\ Shanghai Jiao Tong University, Shanghai 200240, China; \\ ${ }^{4}$ IFSA Collaborative Innovation Center, Shanghai Jiao Tong University, Shanghai 200240, China and \\ ${ }^{5}$ School of Earth and Space Sciences, University of Science and Technology of China, Hefei, 230026, China
}

( Dated: June 21, 2015)

\begin{abstract}
We present the particle-in-cell (PIC) simulation results of the interaction of a high-energy lepton plasma flow with background electron-proton plasma and focus on the acceleration processes of the protons. It is found that the acceleration follows a two-stage process. In the first stage, protons are significantly accelerated transversely (perpendicular to the lepton flow) by the turbulent magnetic field "islands" generated via the strong Weibel-type instabilities. The accelerated protons shows a perfect inverse-power energy spectrum. As the interaction continues, a shockwave structure forms and the protons in front of the shockwave are reflected at twice of the shock speed, resulting in a quasi-monoenergetic peak located near $200 \mathrm{MeV}$ under the simulation parameters. The presented scenario of ion acceleration may be relevant to cosmic-ray generation in some astrophysical environments.
\end{abstract}

Keywords: particle acceleration, Fermi acceleration, collisionless shock, lepton plasma flow

PACS numbers: 47.75.+f, 52.35.-g, 52.65.-y, 98.70.Sa

\section{INTRODUCTION}

The origin of high-energy cosmic-rays over a wide range of energies and the non-thermal emission of radiation from a wide variety of high energy astrophysical sources is the most fundamental problem in astrophysics and has been studied for over six decades since Teller and Fermi[1], however, it has not been fully resolved till now. Usually these radiations are attributed to mechanisms like inverse Compton scattering $[2,3]$ and/or synchrotron emission[4] from high energy particles and the problem turns to how these power-spectrum energetic particles are generated. Considerable efforts are devoted to finding possible generation mechanisms of such energetic particles[5, 6]. As far back as 1949, Fermi purposed the stochastic acceleration mechanism suggesting that the particles can be accelerated through the collision with magnetic "islands" in the space[1]. Recently, Hoshino presented a similar process using a magnetic reconnection configuration in pair plasmas [7]. Another hopeful candidate of "cosmic particle accelerator" is the collisionless shock. It is believed to exist widely in the interstellar space and play an important role in supernova remnants (SNRs) $[8,10]$, jets of radio galaxies [9], gamma-ray bursts (GRBs)[11, 12] and the formation of the large scale structure of the Universe[13, 14]. Therefore, it has been modelled by plenty of researchers both analytically [15] and numerically[16, 17].

Although both stochastic acceleration and collisionless shock acceleration have been well modelled separately in lots of publications, most of them are restricted to the phenomena themselves. Presumed particular initial conditions are used to ensure the occurrence of the interested phenomenon. For example, in Hoshino's work [7], four Harris current sheets are imposed to build magnetic islands; in the collisionless shock simulations[1619], a reflecting boundary condition is used to "make" the shock. These studies have significantly improved our general understanding of the stochastic acceleration and shock dynamics. However, more realistic modelling in a wide parameter range is still needed.

In this work, we present a scenario of proton acceleration processes in two distinct processes occurring naturally during the interaction of a high-energy lepton plasma flow or jet with background electron-proton plasma. This system of interaction corresponds to several astrophysical models such as the pulsar-wind shock system[20], the fireball model of GRBs[21] and the recently purposed Binary orbits model of novae $\gamma$-ray emission[22]. Also the electron-positron jets are found in different astrophysical environments such as quasars [23], black holes [24], and so on. Without using any artificial setup to facilitate any specific acceleration process, by use of the particle-in-cell (PIC) simulation, we find that the background protons in the system are naturally accelerated via Fermi acceleration like processes. The acceleration can be divided into two stages which are dominated by different mechanisms [25]. In the early stage, stochastic acceleration, which is driven by turbulent magnetic fields generated by strong Weibel-type instabilities, dominates and the protons are accelerated transversely. As time elapses, a collisionless shock gradually forms in the background plasma, which accelerates the protons 
longitudinally. These results provide a clear physics picture of a high-energy plasma flow (jet, pulsar-wind, and so on) interaction with interstellar masses and thus can improve our understanding of the related astrophysical phenomena.

\section{SIMULATION SETUP}

The numerical simulation was performed by our selfencoded two-dimensional PIC code KLAPS [26]. We simulate a two-dimensional unmagnetized system with the electron-ion background plasma which homogeneously fills the whole simulation domain located at $(x, y) \in\left[0,900 d_{e}\right] \times\left[0,95 d_{e}\right]$, where $d_{e}=c / \omega_{p e}=$ $\left(4 e^{2} n_{p} / m_{e} c^{2}\right)^{-1 / 2}$ is the election skin depth for a number density $n_{p}$ and electron mass $m_{e} ; e$ and $c$ here denote the elementary charge and the speed of light in vacuum, respectively. We use a real ion-electron mass ratio in the simulation, i.e., $m_{i} / m_{e}=1836$. The initial temperature for background plasma is $500 \mathrm{eV}$. A monoenergetic pair plasma flow consists of electrons and positrons which are injected along $+x$ direction from the left boundary of the simulation domain with initial energy $\sim 50 \mathrm{MeV}\left(\gamma_{e^{+}, e^{-}}=100\right)$ and interact with the background plasma. The initial density of the flow $n_{f}$ is equal to $n_{p}$. The whole system is numerically resolved with 8 cells per $d_{e}$ in both directions with 9 particles per cell for each species, thus ensuring that the behavior of leptons can be modelled accurately. The time resolution of the simulation is $0.06 \omega_{p e}^{-1}$. An open boundary condition is used for $x$-direction and a periodic boundary condition for $y$-direction.

\section{RESULTS AND DISCUSSIONS}

First stage: stochastic acceleration

In the beginning, the flow is uniform along the $y$ direction. However, due to the relative motion between the lepton flow and background, Weibel-type instabilities can occur and generate filamentous structures at $x<100 d_{e}$ after several hundreds of $1 / \omega_{p e}$. These filaments make the newly incoming flow leptons filamented, as shown in Figure 1(a)-(b). This filamented high-energy lepton plasma flow then propagates deeper and causes a highly turbulent region at $100-400 d_{e}$ after about $900 \omega_{p e}^{-1}$. Within the turbulent region, the density of ions can vary more than three orders of the magnitude, as shown in Figure 1(c). Such strong turbulence can generate strong magnetic fields which are displayed in Panel (d) of Figure 1 [27]. As said before, the paper mainly focuses on the acceleration of the ions in the background plasma (referred as "ions" after for simple). Figures 1(e)-(g) gives the phasespace distribution of the ions. The ions are ac-
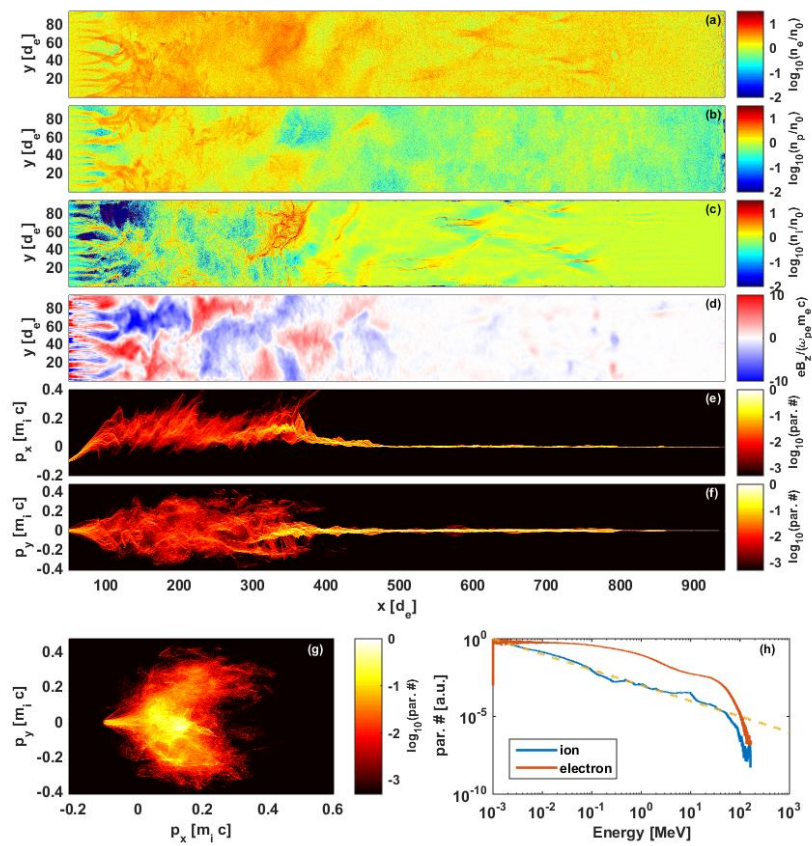

FIG. 1. The space distributions of (a)electrons, (b)positrons, (c)ions and (d)magnetic fields as well as (e) $x-p_{x}$, (f) $x-p_{y}$ and (g) $p_{x}-p_{y}$ projections of the ion's phasespace distribution at $t=950 \omega_{p e}^{-1}$. (h) shows the energy spectrum of background ions (blue line) and electrons (orange line) at the same time, with the line $\sim E^{-1}$ (dashed line).

celerated in both $x$ and $y$ directions. The longitudinal acceleration in $x$ direction can be simply attributed to the momentum transfer between the flow leptons and the background particles through the electromagnetic fields. However, it can be seen from Figure 1(g) that the transverse acceleration is even stronger than the longitudinal acceleration. Moreover, the accelerated protons forms a perfect inverse-power energy spectrum

$$
d N / d E \sim E^{-1}
$$

with the cutoff energy near $100 \mathrm{MeV}$, as shown in Figure $1(\mathrm{~h})$.

To interpret the strong transverse acceleration and the inverse-power energy spectrum, we notice the strong turbulence-generated magnetic fields. Fermi demonstrates that particles can be accelerated by collisions with the moving magnetic islands, and proves that for a large number of collisions, the resulted energy spectrum will be in the form of $d N / d E \sim E^{-\left[1+\tau /\left(B^{2} T\right)\right]}[1]$, where $\tau$ is the average time between two collisions and $T$ is the lifetime of the particle before being absorbed or escaped from the region. This is often referred to as Fermi II acceleration. Consider the following fact that (1) our code for this simulation does not include the annihilate or recombination module, i.e., the local particle number conserves, (2) a periodic boundary condition is used in $y$-direction, and (3) the $x$-direction size of the simulation domain is much 

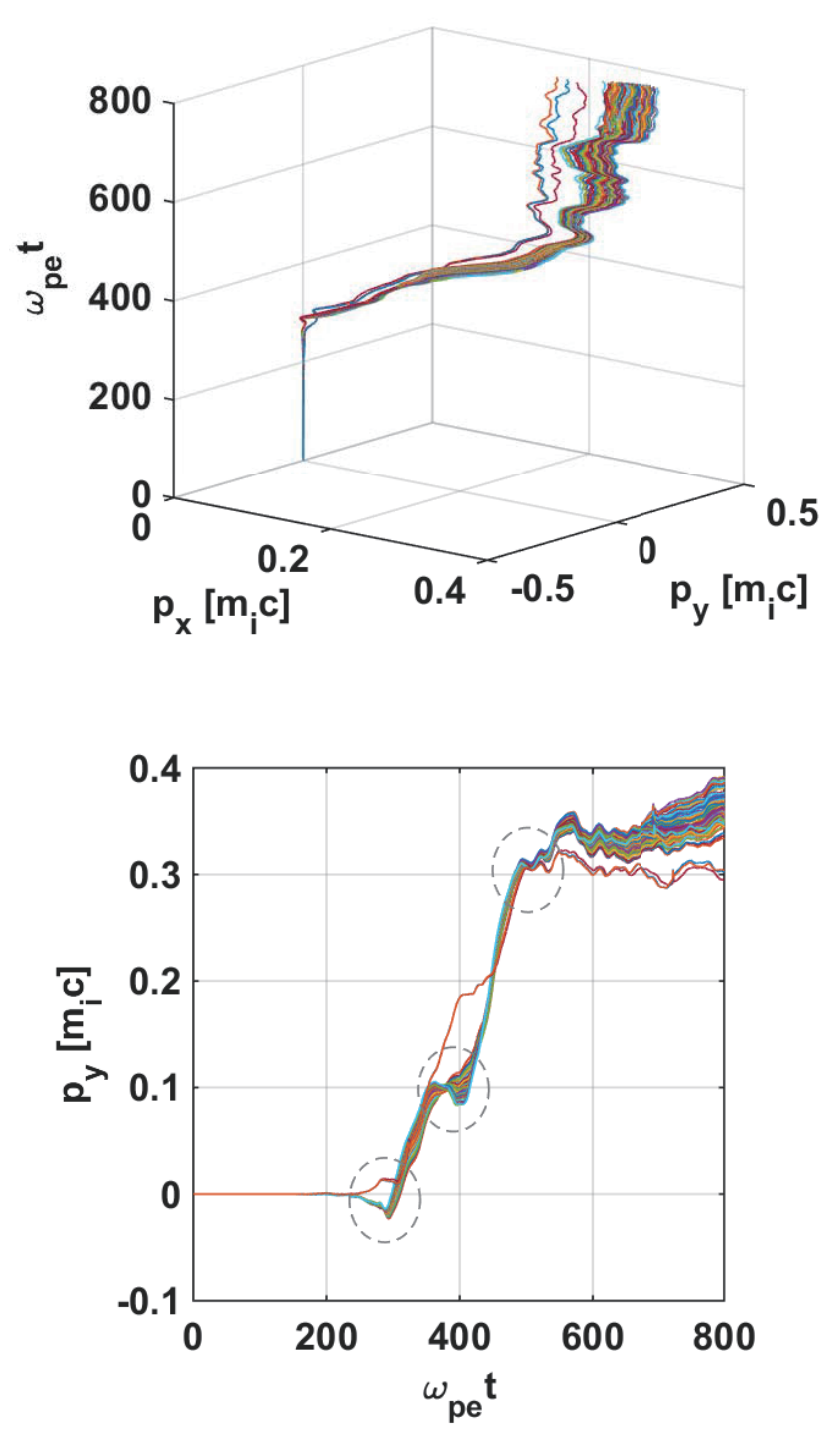

FIG. 2. The momentum space trajectory of 100 ions from background plasma with the largest transverse momentum at $t=950 \omega_{p e}^{-1}$.

larger for ions initially located at $x<400 d_{e}$ to escape. It is thus reasonable to assume that $T \rightarrow \infty$ and then $d N / d E \sim E^{-1}$, which is exactly the result we get in the simulation. We also trace the trajectories of the 100 ions in momentum space with the largest transverse momentum at $t=950 \omega_{p e}^{-1}$, as plotted in Figure 2. One can see obvious evidence of the reflection between significant acceleration or deceleration marked by the grey circles, which indicate that some ions experience sequential acceleration occurs at different locations. This is different from the usual acceleration mechanisms of ions found in relativistic laser-solid interaction in laboratory [28].

We note that some researchers have reported some results from similar models with much smaller incident flow energy $\left(\gamma_{\text {flow }}<10\right)$ since one decade ago and have
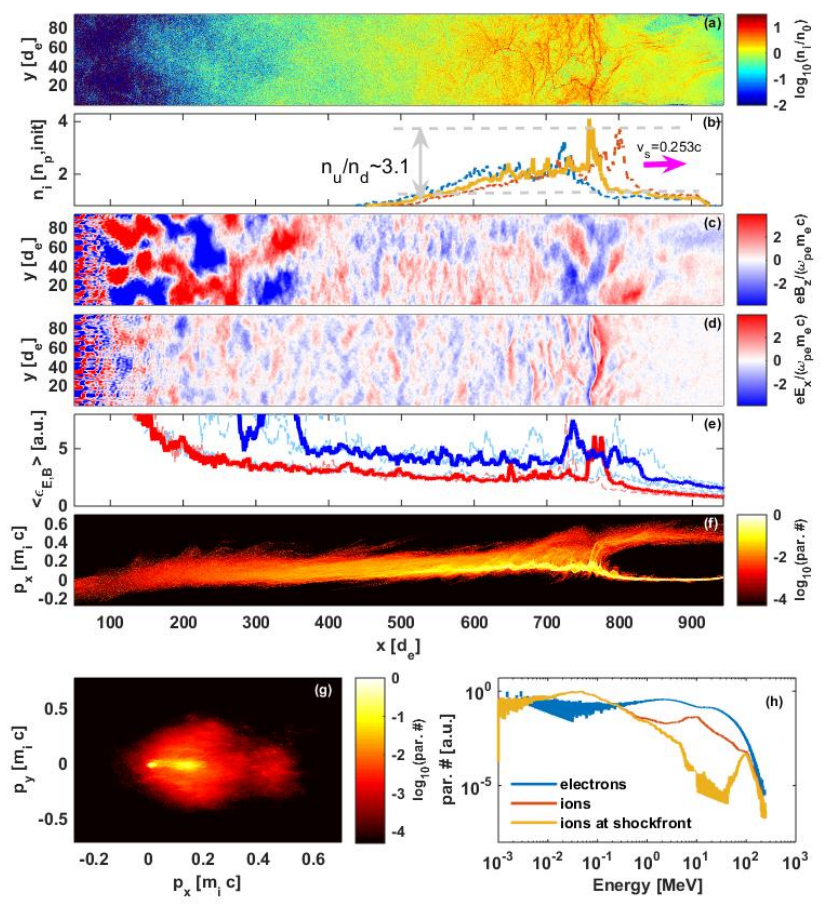

FIG. 3. (a) The space distribution of ion density, (b) the transverse averaged ion density, the space distribution of (c) magnetic field $B_{z}$ and (d) electric field $E_{x}$, (e) transverse averaged field energy densities, blue for magnetic field $B_{z}$ and red for electric field $E_{x}$, (f) $x-p_{x}$ and (g) $p_{x}-p_{y}$ projections of the ions' phasespace distribution and the spectra of background particles at time $t=2500 \omega_{p e}^{-1}$. In panel (b) and (e), the dash lines shows the corresponding quantity at $t=2470 \omega_{p e}^{-1}$ and $t=2530 \omega_{p e}^{-1}$ respectively (details are described in the text).

seen some slight broadening of the energy spectra of the background plasma particles. Because the maximum energy of the energetic particles found in the simulations is small, there are controversial explanations of this energy spectrum broadening. Some researchers regard it as an acceleration[29, 30], while others consider it as thermalization [31]. With the continuous incidents of plasma flows with much higher initial energy, our simulation enables the generation of stronger turbulent magnetic fields and shows irrefragable evidence for the existence of particle acceleration.

\section{Second stage: shock acceleration}

Apparently the lepton jet propagates much faster than the ion-acoustic speed of the background plasma, which is estimated as around $v_{a}=\sqrt{\left(\gamma_{a d} T_{p e} / m_{i}\right)} \approx 0.13 c$. Here $\gamma_{a d}$ is the adiabatic coefficient which equals to 2 for a simple particle in a two-dimensional frame. Since the system is highly disequilibrium, $T_{p e}$ is approximated with the average energy of background electrons, which is about $7 \mathrm{MeV}$ according to the simulation. As a result, a shock 
structure gradually forms in the background plasma as the interaction continues. Figure 3(a) shows the ion density distribution at $t=2500 \omega_{p e}^{-1}$ and its transverse average is plotted in Figure 3(b) with the cases of $30 \omega_{p e}^{-1}$ earlier and later (shown by the blue and dark-red dash lines, respectively). One can see clearly that a shock wavefront propagates forward at a speed of $v_{S} \simeq 0.253 c$, which gives a Mach number $M=1.95$. And the density ratio between downstream and upstream of the shock is measured as around $n_{\text {down }} / n_{\text {up }} \sim 3.1$ in Figure 3(b). The shock hydrodynamic jump conditions give

$$
\begin{gathered}
n_{\text {down }} / n_{u p}=\left(\gamma_{a d} \hat{\gamma}+1\right) /\left(\gamma_{a d}-1\right) \\
v_{S}=c\left[\left(1+\gamma_{a d} \hat{\gamma}\right) \hat{p}\right] /\left[1+\hat{\gamma}+\gamma_{a d} \hat{p}^{2}\right]
\end{gathered}
$$

for a well defined shock wave[32, 33], in which $\gamma_{a d}$ is the adiabatic coefficient with value 2 as discussed in the beginning of this paragraph. $\hat{p}$ denotes the relative momentum (normalized by the rest mass multiplying $c$ ) of the downstream flow in the frame of the upstream (which in our case is the rest frame) and $\hat{\gamma}$ is the related Lorentz factor. According to Figure 3(f), we take $\hat{p} \sim 0.17$ as a reasonable estimate, then $\hat{\gamma} \sim 1.014$. Put these values into the above formulas, we obtain $n_{\text {down }} / n_{\text {up }}=3.03$ and $v_{S}=0.248 c$, which agree with the measurement in our simulation.

Panels (c) and (d) of Figure 3 present the distributions of electromagnetic fields. One can see that at $x<400 d_{e}$, the turbulence-generated electromagnetic fields discussed in the earlier part of the paper still remain strong although the ions are almost blown up by the lepton flow. The field intensity dramatically drops to some random fluctuations. However, at the place of the shock wavefront, the fields become strong again and form a peak. Interestingly, we find that the peak positions of the electric and magnetic fields do not overlap with each other. The former locates in front of the wavefront; the latter locates behind the wavefront and exists in a broader range, which is because the sources of the fields are different. The electric field is generated by the charge separation in front of the wave front while the source of the magnetic field is the instabilities. The fields move with the shock and then reflect the ions at the shock front, with a speed of twice of the shock wave speed, as one can confirm through the high branch in Figure 3(f) for the longitudinal phasespace. Figure $3(\mathrm{~g})$ shows the $p_{x}-p_{y}$ projections of the ion's phasespace distribution. There is no further transverse acceleration comparing with $t=950 \omega_{p e}^{-1}$, while the longitudinal acceleration is significantly enhanced by the shock. We also plot the energy spectra of the background electrons and ions in Figure 3(h). The spectrum of ions is not perfectly inverse-power as a whole now, but there are piecewise inverse-power parts at $10^{-1}-10^{1} \mathrm{MeV}$ and $10^{1}-10^{2} \mathrm{MeV}$, which forms a "knee" structure. Similar phenomena are found in astronomical observations as well $[34,35]$. The orange line plots the spectrum of ions

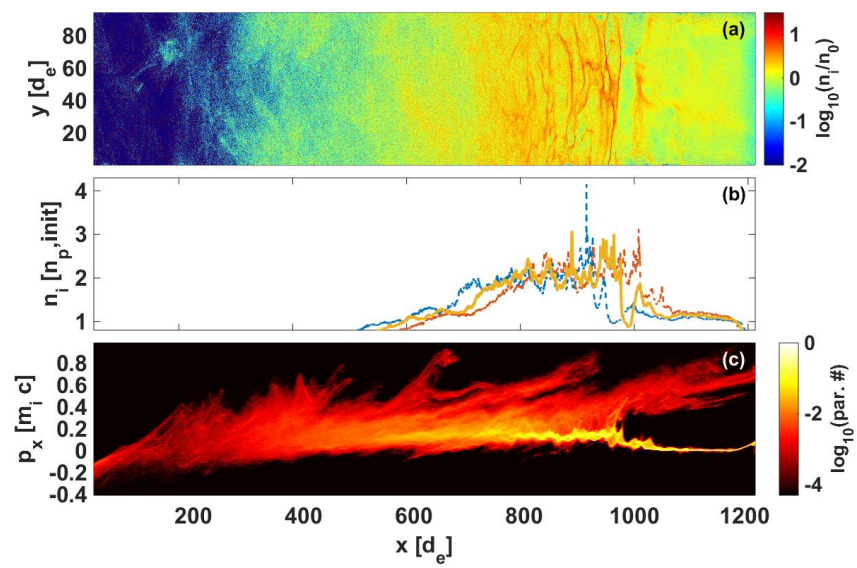

FIG. 4. (a) The space distribution of the background ion density, (b) the transverse averaged ion density and (c) $x-p_{x}$ projections of the ions' phasespace distribution at time $t=$ $2500 \omega_{p e}^{-1}$ for the initial $100 \mathrm{MeV}$ lepton flow case.

located at $x>800 d_{e}$ (shockfront). It shows that a quasimonoenergetic peak is formed by the shock-reflected ions at about $200 \mathrm{MeV}$, which is four times larger than the energy of the injecting lepton flow. Here we would like not to discuss the details of shock acceleration since it is well studied in plenty of previous publications[16-19].

To check the robustness of the above ion acceleration processes, we have also performed another simulation with higher initial energy of the lepton flow of $\sim 100 \mathrm{MeV}\left(\gamma_{e^{+}, e^{-}}=200\right)$ with other conditions unchanged. Similar phenomena are observed. In the first stage, the protons, which are accelerated by the Fermi II mechanism as well as momentum transfer from the lepton flow longitudinally, form a perfect inverse-power spectrum. The cut-off energy is slightly increased to $\sim 130 \mathrm{MeV}$. However, this increase is caused only by the increase of longitudinal momentum, which implies that the Fermi II mechanism is not enhanced, i.e., the average speed of the "magnetic islands" generated by the instabilities is insensitive to the initial energy of the flow. On the other hand, a lepton flow with higher energy can provide more longitudinal momentum for the downstream electrons, which significantly increases its temperature to $15.7 \mathrm{MeV}$. As a result, the ion-acoustic speed increases to $0.18 c$ and the measured shockwave speed increases to $v_{S}=0.293 c$. The ions reflected by this shock wave form a quasi-monoenergetic structure at about $100-400 \mathrm{MeV}$. However, for this $\gamma_{e^{+}, e^{-}}=200$ case, we observe that some ions in the downstream also have very high energy despite their small population, as shown in Figure 4(c), which is slightly different from the $\gamma_{e^{+}, e^{-}}=100$ case. This may lead to some injected acceleration into the shock from the pre-accelerated ions in the downstream region. We also present the space distribution of the ion 
density in Figures 4(a) and 4(b) for comparison with the corresponding panels in Figure 3.

\section{CONCLUSION}

In conclusion, we have studied the interaction of a high-energy lepton flow with a background normal ionelectron plasma via PIC simulation. A scenario of twostage acceleration of the background ions is identified: In the first stage, the main mechanism is type-II Fermi acceleration found both in the transverse and longitudinal directions. The accelerated ions forms a globally inversepower energy spectrum. As time passes, the acceleration gradually enters the second stage as a shock wavefront forms in the background plasma. The shock propagates in the longitudinal direction with a Mach number of about 2, which further accelerates the background ions to energy higher than that gained in the first stage. While the ion spectrum is no longer globally inverse-power in the second stage, it becomes piecewise inverse-power with a knee structure. And the results, which will be relevant with the interaction between cosmic flows with interstellar matters, are helpful to explain the origin of the cosmic rays with inverse-power spectrum. The acceleration scenario presented in this work may be tested experimentally in the future as the high energy lepton flows can be created nowadays by high power lasers [36-38].

This work was partially supported by the National Basic Research Program of China (Grant No. 2013CBA01500), and the National Science Foundation of China (Grant Nos. 11421064, 11220101002, 11129503, 11135012).

*zmsheng@sjtu.edu.cn

[1] Fermi E. On the origin of the cosmic radiation. Phys. Rev., 1949, 75:1169-1174

[2] Aharonian F et al. Discovery of the two wings of the kookaburra complex in vhe $\gamma$-rays with hess. Astronomy \& Astrophys, 2006, 456:245-251

[3] Aharonian F et al. First detection of a vhe gamma-ray spectral maximum from a cosmic source: Hess discovery of the vela $\mathrm{x}$ nebula. Astronomy \& Astrophys, 2006, 448:L43-L47

[4] Ginzburg V L and Syrovatsk S I. Developments in the theory of synchrotron radiation and its reabsorption. Ann Rev Astrono \& Astrophys, 1969, 7:375-420

[5] Bhattacharjee P and Sigl G. Origin and propagation of extremely high-energy cosmic rays. Phys Rep, 2000, 327:109-247

[6] Olinto A V. Ultra high energy cosmic rays: the theoretical challenge. Phys Rep, 2000, 333C334:329-348

[7] Hoshino M. Stochastic particle acceleration in multiple magnetic islands during reconnection. Phys Rev Lett, 2012, 108:135003
[8] Blandford R and Eichler D. Particle acceleration at astrophysical shocks: A theory of cosmic ray origin. Phys Rep, 1987, 154:1-75

[9] Begelman M C, Rees M J, and Sikora M. Energetic and radiative constraints on highly relativistic jets. Astrophys J, 1994,429:L57-L60

[10] Wang B, Yuan Q, Fan C, et al. A study on the sharp knee and fine structures of cosmic ray spectra Sci China - Phys Mech Astron, 2010, 53:842-847

[11] Zhang B and Meszaros P. Gamma-ray bursts: Progress, problems \& prospects. Int J Mod Phys A, 2004, 19:23852472

[12] Piran T. The physics of gamma-ray bursts. Rev Mod Phys, 2005,76:1143-1210

[13] Loeb A and Waxman E. Cosmic [gamma]-ray background from structure formation in the intergalactic medium. Nature, 2000, 405:156-158

[14] Gruzinov A. Gamma-ray burst phenomenology, shock dynamo, and the first magnetic fields. Astrophys J Lett, 2001, 563:L15-L18

[15] Ucer D and Shapiro V D. Unlimited relativistic shock surfing acceleration. Phys Rev Lett, 2001, 87:075001

[16] Haugbølle T. Three-dimensional modeling of relativistic collisionless ion-electron shocks. Astrophys J Lett, 2011, 739(2):L42-L45.

[17] Martins S F, Fonseca R A, Silva L O, and Mori W B. Ion dynamics and acceleration in relativistic shocks. Astrophys J Lett, 2009, 695:L189-L192

[18] Amato E and Arons J. Heating and nonthermal particle acceleration in relativistic, transverse magnetosonic shock waves in proton-electron-positron plasmas. Astrophys J, 2006, 653:325-338

[19] Yang Z Y, Lu Q M, Lembège B, and Wang S. Shock front nonstationarity and ion acceleration in supercritical perpendicular shocks. J Geophys Res: Space Physics, 2009, 114(A3):2156-2202

[20] van der Swaluw E, Achterberg A, Gallant Y A, Downes $\mathrm{T} \mathrm{P}$, and Keppens R. Interaction of high-velocity pulsars with supernova remnant shells. Astronomy \& Astrophys, 2003, 397:913-920

[21] Waxman E. Gamma-ray bursts and collisionless shocks. Plasma Phys Control Fusion, 2006, 48:B137

[22] Chomiuk L, Linford J D, Yang J, et al. Binary orbits as the driver of [ggr]-ray emission and mass ejection in classical novae. Nature, 2014, 514:339-342

[23] Wardle J F C, Homan D C, Ojha R and Roberts D H, ElectronCpositron jets associated with the quasar $3 \mathrm{C} 279$, Nature, 1998, 395: 457-461

[24] Ruffini R, Vereshchagin G, Xue S S, ElectronCpositron pairs in physics and astrophysics: From heavy nuclei to black holes, Phys Rep, 2010, 487: 1-140

[25] Cui Y Q, Numerical studies on selected problems in the interaction of intense lasers/particle beams with bulk plasma, PhD Thesis, University of Chinese Academy of Sciences, May 2015

[26] Wang W M, Gibbon P, Sheng Z M, and Li Y T. Integrated simulation approach for laser-driven fast ignition. Phys Rev E, 2015, 91:013101

[27] Mondal S, Narayanan V, Ding W J, Lad A D, Hao B, Ahmad S, Wang W M, Sheng Z M, Sengupta S, Kaw P, Das A, and Kumar G R. Direct observation of turbulent magnetic fields in hot, dense laser produced plasmas. Proc Nat Acad Sci, 2012, 109:8011-8015 
[28] Su L N, et al. Proton angular distribution research by a new angle-resolved proton energy spectrometer Sci China - Phys Mech Astron, 2014, 57:844-848

[29] Silva L O, Fonseca R A, Tonge J W, et al. Interpenetrating plasma shells: Near-equipartition magnetic field generation and nonthermal particle acceleration. Astrophys J, 2003, 596:L121-L124

[30] Nishikawa K I, Hardee P, Richardson G, Preece R, Sol H, and Fishman G J. Particle acceleration in relativistic jets due to weibel instability. Astrophys J, 2003, 595:555-563

[31] Frederiksen J T, Hededal C B, Haugbø lle T, and Nordlund A. Magnetic field generation in collisionless shocks: Pattern growth and transport. Astrophys J, 2004, 608:L13

[32] Blandford R D and McKee C F. Fluid dynamics of relativistic blast waves. Phys Fluids, 1976, 19:1130-1138

[33] Fiuza F, Fonseca R A, Tonge J, Mori W B, and Silva L O. Weibel-instability-mediated collisionless shocks in the laboratory with ultraintense lasers. Phys Rev Lett, 2012,
108:235004

[34] Ter-Antonyan S. Sharp knee phenomenon of primary cosmic ray energy spectrum. Phys Rev D, 2014, 89:123003

[35] Garyaka A P, Martirosov R M, Ter-Antonyan S V, Nikolskaya N, Gallant Y A, Jones L, and Procureur J. Rigiditydependent cosmic ray energy spectra in the knee region obtained with the gamma experiment. Astroparticle Phys, 2007, 28:169-181

[36] Gahn C, Tsakiris G D, Pretzler G, et al. Generating positrons with femtosecond-laser pulses, Appl Phys Lett, 2000, 77: 2662-2664

[37] Chen H, Wilks S C, Meyerhofer D D, et al. Relativistic quasimonoenergetic positron jets from intense laser-solid interactions, Phys Rev Lett, 2010, 105: 015003

[38] Sarri G, Poder K, Cole J M, et al. Generation of neutral and high-density electronCpositron pair plasmas in the laboratory Nature Comm, 2015, 6: 6747 